\title{
The Role of the Endometriosis Fertility Index (EFI) and Endometriosis Scoring Systems in Predicting Infertility Outcomes
}

\author{
Andrew S. Cook • G. David Adamson
}

Published online: 27 June 2013

(C) Springer Science+Business Media New York 2013

\begin{abstract}
Many aspects of endometriosis continue to make it an enigmatic medical condition nearly a century after its first description as a specific disease. The Endometriosis Fertility Index (EFI) is the first validated scoring system that is predictive of nonassisted reproductive technologies pregnancy following surgical evaluation when the patient has functional gametes and uterus. Pregnancy rates decrease significantly in patients with decreased ovarian reserve, which is especially a problem in older patients and also, as reflected in the EFI, decrease over time if pregnancy does not occur in the first year or two following surgery. Patient management algorithms using this information to assist in deciding when to continue nonassisted reproductive technologies vs. assisted reproductive technologies attempts at conception are presented.
\end{abstract}

Keywords Endometriosis $\cdot$ Fertility $\cdot$ Index $\cdot$ Scoring $\cdot$ Prediction

\section{Introduction}

Many aspects of endometriosis continue to make it an enigmatic medical condition nearly a century after its first description as a specific disease. Our suboptimal understanding of this disease

\footnotetext{
A. S. Cook

Vital Health Institute, 14830 Los Gatos Blvd.,

Suite 300, Los Gatos, CA 95032, USA

e-mail: acook@vitalhealth.com

G. D. Adamson $(\bowtie)$

Fertility Physicians of Northern California,

540 University Ave., Suite 200, Palo Alto,

CA 94301, USA

e-mail: gdadamson@arcfertility.com
}

compromises many aspects of patient care and quality of life, resulting in inefficient care and increased direct and economic costs of endometriosis.

The primary presenting symptoms of endometriosis are infertility and/or pain. These, along with many other symptoms (e.g., fatigue), can combine to reduce quality of life, productivity, and even the ability to function with basic daily tasks.

Several basic issues need to be elucidated to facilitate more efficient and effective care. These include improved diagnosis and staging of endometriosis. A simple-to-use, sensitive and accurate, noninvasive diagnostic test is a critical component of providing improved and more timely care to women with endometriosis. Discussion of improved diagnostic testing is beyond the scope of this chapter. This chapter is focused on recent clinical advances in the ability to predict endometriosis fertility outcomes.

In general, a scoring or staging system is ideally based on scientific data providing both meaningful and consistent results that are easy for the health care provider to understand and utilize. It should provide a measure of the impact of the disease on the patient's functionality, predict future fertility, predict degree of pain relief with any given treatment, and provide an estimate of risk of recurrence of endometriosis.

Historically, none of the endometriosis staging systems was able to predict the chance of conception following treatment until the Endometriosis Fertility Index (EFI) was published in October 2010. The EFI is predictive of nonassisted reproductive technologies pregnancy following surgical evaluation.

Since publication of the EFI, recent studies continue to provide additional information about factors that are helpful to expand our understanding from which a comprehensive fertility treatment plan can be created. This paper will review this information and present an evidence-based/linked fertility treatment algorithm. 


\section{Ovarian Reserve, Oocyte Quality, and Age}

Fundamentally both a viable male and female gamete is required for conception. Decreased ovarian reserve (DOR) is reflective of a decreasing quantity and quality of oocytes, embryos at assisted reproductive technologies (ART), and resulting fertility. In fact, DOR is currently the second leading cause of infertility [1]. DOR can be a result of a variety of factors but is most commonly seen with advanced maternal age. Age of the female partner is one of the most important factors influencing fertility [2•]. Women who are older than 35 years and have not conceived in 6 months should undergo expedited evaluation and treatment [3-5]. Because decreasing quality of female gametes and ability to result in successful pregnancy is irreversible, its status dictates much of the treatment algorithm for fertility patients. Historically, reproductive success often has been a result of repeated exposure rather than efficiency. Successful fertility treatment often is a combination of increasing monthly fecundity along with repeated attempts at pregnancy. Unfortunately, couples presenting with infertility issues often are at an age where DOR is or will soon become a significant factor in their treatment.

The ASRM recently has produced a document that addresses the complexity of DOR [6••]. Currently, there is no uniformly accepted definition of DOR, as the term may refer to three related but distinctly different outcomes: oocyte quality, oocyte quantity, or reproductive potential. The document did not address endometriosis specifically, and it may be that endometriosis patients have both similarities and differences with respect to the populations described in the studies used to reach the summary and concluding statements. This document summarized that available evidence concerning the performance of ovarian reserve tests is very limited by small sample sizes and performance of the studies such that the study results cannot easily be applied in clinical practice. A screening test itself can have very different outcomes depending on sensitivity and specificity characteristics and cannot diagnose decreased ovarian reserve. Overall, follicle stimulating hormone (FSH) is the most commonly used screening test for DOR, but antral follicle count (AFC) and anti-müllerian hormone (AMH) are promising predictors. Home tests of ovarian reserve have serious limitations and easily can provide false reassurance or unnecessary anxiety and concern.

The document concluded that there is insufficient evidence to recommend that any ovarian reserve test now available should be used as the sole criterion for use of ART. The number of false-positive test results will increase when screening tests for decreased ovarian reserve are used in low-risk populations. (It is not known whether endometriosis patients are low risk or high risk, but those with prior ovarian surgery are likely in higher-risk populations.) There is fair evidence to indicate that FSH has high specificity, but low sensitivity, when a high cutpoint value is used for predicting poor response to ovarian stimulation or failure to conceive. There is fair evidence to refute the notion that ovarian response or pregnancy rates will be improved in cycles in which the FSH concentration is normal among women previously exhibiting abnormally elevated values. There is fair evidence that the basal estradiol concentration helps in the accurate interpretation of basal FSH concentrations used to screen for decreased ovarian reserve. There is reasonable evidence to suggest that a clomiphene citrate challenge test has mildly increased sensitivity to detect decreased ovarian reserve compared with basal FSH concentration. There is mounting evidence to support the use of AMH as a screening test for poor ovarian response, but more data are needed. There is emerging evidence to suggest that a low AMH level (e.g., undetectable AMH) has high specificity as a screen for poor ovarian response but insufficient evidence to suggest its use to screen for failure to conceive. There is fair evidence to support that a low antral follicle count (3-10) has moderate to high specificity as a screening test for poor ovarian response and insufficient evidence to support the use of AFC as a screening test for failure to conceive. There is insufficient evidence to indicate that the combined results of multiple screening tests for diminished ovarian reserve are more useful than that of each test alone.

Given our current knowledge, a woman with rAFS stage III-IV endometriosis, especially those with a history of endometrioma, should probably undergo ovarian reserve testing, even at a young age [7]. An argument also can be made for interval evaluation of women in their early to mid 30 s who are delaying pregnancy until age 35 to 40 years [8]. Perhaps future studies will provide data about when we can predict the end of an individual woman's optimal fertility window years in advance, but current studies do not allow this. Patient outcome and the overall economic burden of fertility treatment would be significantly improved if patients had the necessary information to pursue pregnancy proactively before entering into DOR.

\section{Other Potential Issues Contributing to Infertility}

In the uterus, congenital abnormalities, myomas, adenomyosis, polyps, and adhesions can contribute to reduced fertility depending on the severity of the condition. Unfortunately, while severe congenital abnormalities, especially those with a small uterine cavity, or a uterine septum, are thought to reduce fertility, good studies to predict the impact of such conditions are not available. Similarly, while large myomas distorting the uterine cavity likely reduce fertility, the size, number, location, and impact of intramural myomas are not clearly defined. Severe adenomyosis likely reduces fertility, but studies proving 
this are lacking. Polyps can almost certainly be problematic, but the size and location that impact pregnancy are unknown. Minimal intrauterine adhesions may have some impact on pregnancy, whereas moderate and severe adhesions certainly reduce the ability to conceive and have a successful pregnancy. Therefore, these lesions should likely be treated hysteroscopically in some patients who have endometriosis, but patient selection and the degree of improved outcome are difficult to assess [9-12].

The presence of hydrosalpinges makes pregnancy essentially impossible naturally, and salpingostomy carries pregnancy rates of only $5-30 \%$, with an average live birth rate probably less than $20 \%$. Additionally, hydrosalpinges reduce IVF pregnancy rates by approximately half. Therefore, tubal surgery for hydrosalpinges may occasionally be indicated in a favorable prognosis younger patient with limited access to ART or for the patient undergoing ART who should have salpingectomy or proximal tubal occlusion $[13,14 \bullet \cdot]$.

With respect to the male, a normal semen analysis is one of the major factors to predict a better prognosis of conception. The impact of only a fair semen analysis may be ameliorated by attention to weight, medications, toxicants, heat, varicoceles, and other health factors. Intrauterine insemination may be of limited benefit in some patients. Overall, for severe male factor, IVF often is the only biologic option, with donor insemination a potential alternative. Men with semen abnormalities should be referred to an expert in male infertility $[15,16]$. Other factors can affect female fertility. Obesity is a major problem in many countries, both developed and developing. The primary underlying factor affecting fertility in obese women is ovulatory dysfunction $[17,18]$.

Cigarette smoking substantially affects fecundity and reproduction. Reported effects include conception delay, accelerated follicular depletion, a variety of effects on sperm parameters and increased miscarriage rates [19]. Other systemic disease, such as diabetes mellitus or thyroid abnormalities, should be managed appropriately.

\section{Surgery vs. ART for Endometriosis-Related Infertility}

The highest pregnancy rates for women with endometriosisrelated infertility are often achieved with a combination of treatments utilizing both surgery and ART. This combined treatment approach can provide significantly higher pregnancy rates than either surgery or ART alone. Of the 825 patients in a large study, 483 underwent surgery as the primary option; 262 became pregnant $(54.2 \%)$. Of the surgery patients who did not conceive, 144 underwent ART, resulting in 56 pregnancies $(30.4 \%)$. The 318 combined total pregnancies from surgery and ART resulted in an overall pregnancy rate of $65.8 \%$. Sixty-eight of the 173 patients undergoing ART as the first treatment option achieved pregnancy $(32.2 \%)$. Spontaneous pregnancy occurred in 20 of the 169 patients $(11.8 \%)$ who received no treatment. The mean time to pregnancy for the surgery patients in this study was 11.2 months [20•].

Medical treatments are not indicated, either alone or following surgery, for treatment of endometriosis-related infertility. The role and timing of surgery for infertility patients with ovarian endometriomas is controversial. There is a delicate balance between the benefits of endometriomectomy, which have not been proven, and the possibility of causing decreased ovarian reserve as a result of injury during removal of the endometrioma. ART as a primary treatment option is generally indicated in patients with decreased ovarian reserve and bilateral ovarian endometriomas. The presence of pelvic pain, hydrosalpinges, and very large endometriomas may require surgery before ART. Patients who initially pursue ART but are unsuccessful in achieving pregnancy should consider undergoing surgery for treatment of their endometriosis to improve their chance of conception spontaneously or with the aid of additional ART. Generally, it is preferable to perform surgery first, if clinically indicated, and to perform ART if pregnancy does not occur after 9-15 months. There are very few patients for whom ART should be chosen as the first treatment with a plan to follow with surgery if the ART is unsuccessful.

\section{The Endometriosis Fertility Index Background}

Adamson and Pasta published the first validated and predictive endometriosis staging system in October 2010: the Endometriosis Fertility Index (Fig. 1) [21••]. The approach used to create this system was different than previous systems. A comprehensive statistical analysis of prospectively collected data from a large number of patients utilizing an outcome assessment for fertility revealed which variables were predictive of pregnancy. This methodology was distinctly different from the approach used in creation of previous endometriosis staging systems in which the authors made assumptions of different variables usually based upon surgical observations of various disease-related anatomic changes.

The EFI was externally validated in a recent study [22]. The relationship between the EFI and non-ART pregnancy was felt to be highly significant. The predictive accuracy and discriminative performance was moderate. Future studies may find additional variables not currently included in the EFI that are predictive of pregnancy. Refinement of the EFI over time based on well-designed studies validating the benefit of adding additional variables to the EFI may help further increase the accuracy and discriminative performance of this staging system. 


\section{ENDOMETRIOSIS FERTILITY INDEX (EFI) SURGERY FORM}

\section{LEAST FUNCTION (LF) SCORE AT CONCLUSION OF SURGERY}

\begin{tabular}{|c|c|c|c|c|c|c|c|}
\hline Score & Description & & Left & & Right & & \\
\hline 4 & $=$ Normal & Fallopian Tube & & & & & \\
\hline 3 & $=$ Mild Dyatunction & & & & & & \\
\hline 2 & $=$ Moderate Dyafunction & Fimbria & & & & & \\
\hline 1 & $=$ Severe Dyafunction & & & & & & \\
\hline 0 & $=$ Absent or Nonfunctional & Ovary & & & & & \\
\hline \multicolumn{2}{|c|}{$\begin{array}{l}\text { To calculate the LF score, add together the lowest score for } \\
\text { the left side and the lowest score for the right aide. If an ovary } \\
\text { is abeent on one side, the LF score is obtained by doubling the } \\
\text { lowest score on the aide with the ovary. }\end{array}$} & Lowest Score & Left & + & Right & $=$ & LF Score \\
\hline
\end{tabular}

ENDOMETRIOSIS FERTILITY INDEX (EFI)

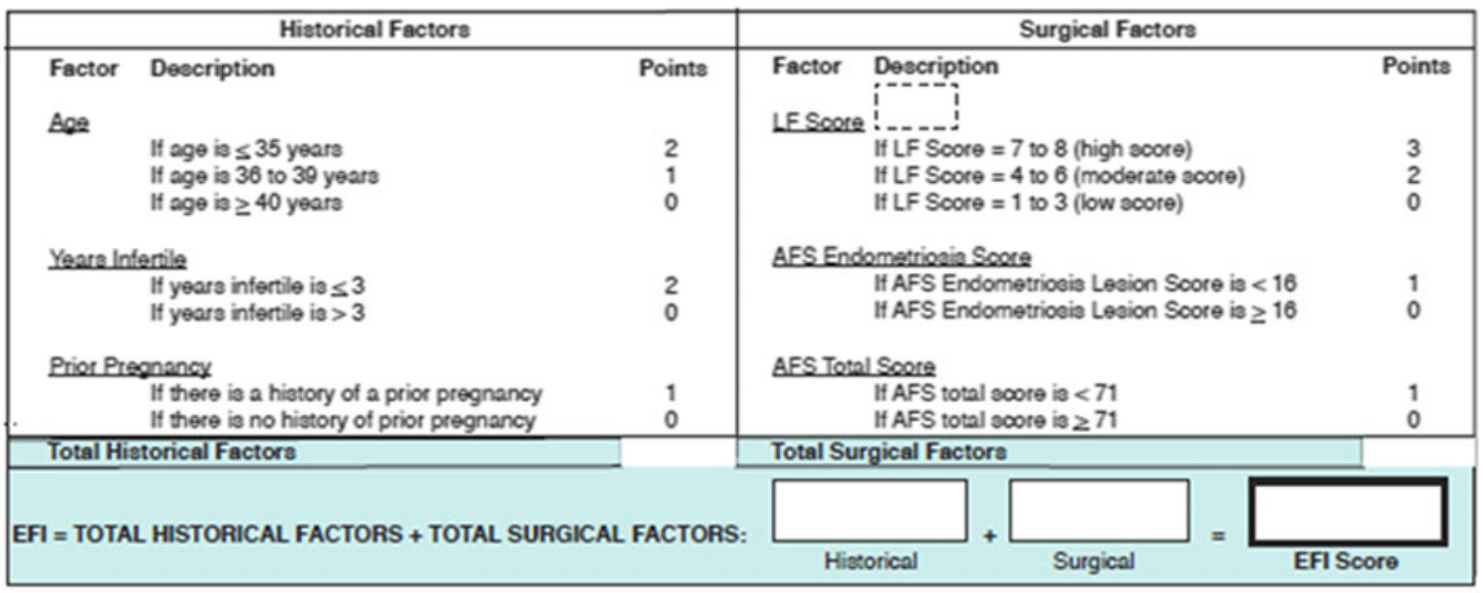

ESTIMATED PERCENT PREGNANT BY EFI SCORE

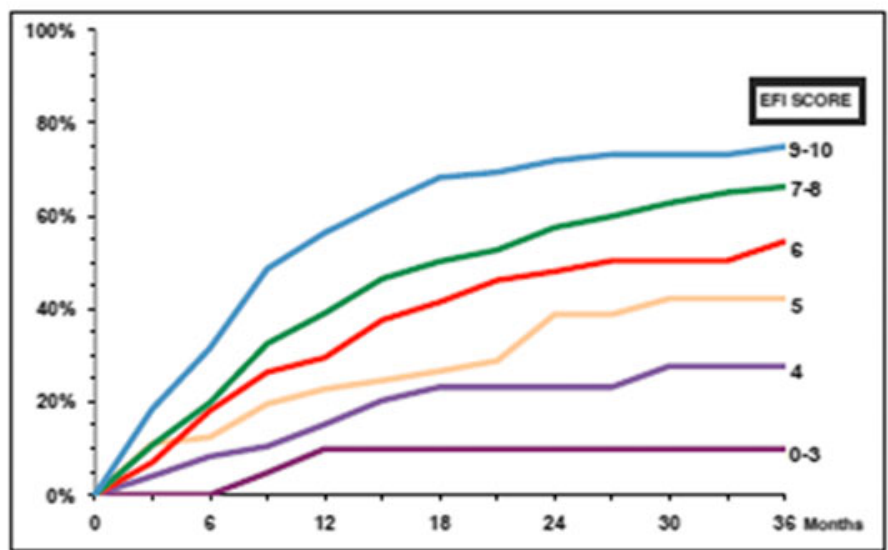

A study of 350 endometriosis associated infertility patients following laparoscopic treatment confirmed the relationship between the Endometriosis Fertility Index and estimated cumulative pregnancy rates [23]. Yacoub et al. studied whether the EFI is a good tool to predict pregnancy in patients with surgically documented endometriosis followed by intrauterine 
insemination (IUI) or in vitro fertilization (IVF) management [24]. Retrospective intention-to-treat analysis was performed on 132 consecutive infertility patients with an isolated endometriosis diagnosis documented and treated by laparoscopy and then followed by ART management (IUI or IVF). Spontaneous or induced pregnancies obtained after laparoscopy were recorded. Women were excluded if they were older than age 40 years or had ovarian deficiency and those with other infertility factors: sperm abnormalities or tubal nonendometriosis-related dysfunction. Fertility as reflected by cumulative birth rate was studied in relation to the endometriosis stage (defined by rAFS score and EFI). Comparison between different stages was assessed using the Kaplan-Meier survival method with a log-rank test. Comparison of life-table curves according to EFI stages shows a significant association between the severity of endometriosis (different stages), infertility, and endometriosis and cumulative birth rate $(P=0.0005)$. However, there was no significant association for the AFS score $(P=0.48)$. The authors concluded that this study confirmed that the AFS score is not a good tool to predict pregnancies and seemed to show that the EFI is a simple and reliable tool to predict pregnancy in patients with surgically documented endometriosis followed by IUI or IVF management. The authors concluded, "In our opinion, this score should be a main component in the choice of the postoperative ART management. Prospective studies are needed to confirm our data."

\section{Clinical Role of the EFI to Predict Likelihood of Non-ART Pregnancy}

To date, the EFI is the most scientifically based predictive staging system for endometriosis-related fertility issues. It provides very precise detailed information in a way that no previous staging system has provided. The two issues that most significantly impact the lives of women with endometriosis are those of fertility and pain. There has not been, nor is there, any current staging system for endometriosis-related pain. A comprehensive endometriosis staging system would provide predictive information for both future fertility and pain relief. Development of an endometriosis pain staging system will most likely follow the approach used in development of the EFI. Postsurgical data outcome on more than 700 endometriosis pelvic pain patients has been prospectively collected during the past 10 years by one of the authors (AC). This large database is undergoing in-depth statistical analysis with the hope of identifying predictive variables. Ideally this will provide the basis to predict both prognosis and risk of recurrence. If a pain staging system similar to the Endometriosis Fertility Index is developed, then ideally a "unified" endometriosis staging system would provide the basis for treating both pelvic pain and infertility patients.

Endometriosis can be one of the most complex and challenging conditions that obstetrician/gynecologists encounter in their practice. Medically, it can be a chronic disease transcending many medical specialties while often responding poorly to standard treatments. Stage III and IV endometriosis (rASRM classification system) and deep infiltrating endometriosis, as well as bowel and ureteral endometriosis, often result in some of the most technically challenging surgeries encountered in surgery, and especially in gynecology. Referral to gynecologic surgeons with expertise in difficult endometriosis cases is essential if patients are to receive the appropriate treatment [25].

We present additional information and tables (Tables 1 and 2) that may assist the physician, and possibly some patients, in understanding prognosis after diagnosis of endometriosis at laparoscopy. These data can be used, if desired, in conjunction with the current EFI worksheet. The information contained in the EFI has been further analyzed to provide summary information that may be helpful when communicating with patients and referring physicians. This additional information also is the basis for an algorithm and guidelines that may be helpful in treatment of endometriosis fertility patients (Table 3).

At least every year patients should be reassessed. In fact, most patients in treatment should be assessed every 3 to 6 months and ongoing management discussed and determined based not only on prognosis but other clinical, social, psychological, financial, and other factors. Depending on her age and years of infertility, the patient will experience a reduced pregnancy rate over time for several reasons: more fertile patients tend to conceive earlier so the remaining population is less fertile (consistent with duration of infertility being a strong predictor of fecundity), the patient gets older, and endometriosis may recur. It has been shown that fecundity is fairly constant after surgery for approximately $12-15$ months before it decreases and is generally very low after 2 years [26]. This time factor in outcome is represented in the EFI by the flattening of the life table curve over time, representing lower pregnancy rates as the patient goes longer without conceiving. This decrease has been presented in a table that shows decreasing fecundity over time for all of the EFI scores (Table 3).

The monthly fecundity data provide a rational basis, creating a table with four levels of treatment groups: Level I has the highest fecundity and Level IV has the lowest. These levels can be used for making treatment recommendations for patients, consistent with the idea that chances for pregnancy decrease over time, and start from different baselines depending on the EFI score. The treatment level and thus treatment recommendations for any given EFI score can change up to three levels over time. 
Table 1 Estimated Fecundity Rates during 1, 2, and 3 years by EFI score

\begin{tabular}{|c|c|c|c|c|c|c|}
\hline \multirow{2}{*}{$\begin{array}{l}\text { EFI score } \\
\text { All patients }(n=801)\end{array}$} & \multicolumn{3}{|c|}{ Monthly fecundity (\%) } & \multicolumn{3}{|c|}{ Yearly fecundity (\%) } \\
\hline & Year 1 & Year 2 & Year 3 & Year 1 & Year 2 & Year 3 \\
\hline $0-3$ & 0.8 & 0.0 & 0.0 & 9.9 & 0.0 & 0.0 \\
\hline 4 & 1.3 & 0.8 & 0.5 & 15.2 & 9.4 & 5.9 \\
\hline 5 & 1.9 & 1.7 & 0.5 & 22.8 & 20.7 & 5.6 \\
\hline 6 & 2.5 & 2.2 & 1.0 & 29.5 & 26.2 & 12.5 \\
\hline 7 & 3.1 & 2.7 & 2.3 & 37.4 & 32.6 & 27.5 \\
\hline 8 & 3.4 & 2.3 & 1.1 & 41.0 & 27.3 & 13.5 \\
\hline $9-10$ & 4.7 & 3.0 & 0.9 & 56.4 & 35.6 & 10.7 \\
\hline
\end{tabular}

The tables suggest that for Level I, with a monthly fecundity greater than $3 \%$ (annual fecundity greater than $35 \%$ ), it is inappropriate to utilize invasive or expensive treatment immediately. Indeed, pregnancy still has approximately a $10-30 \%$ chance occurring in the third year after diagnosis for EFI score 6 and higher.

For patients with Level II, pregnancy rates are 25-35\% (monthly fecundity 2-3\%). By the third year, all EFI scores are Level III or IV, except for EFI score 7 patients, which persist at Level II (see discussion below). Therefore, nonART treatments of EFI score 6 or higher for up to 2 years could be reasonable, but probably not for a third year (except EFI score 7).

For treatment Level III, chance of pregnancy is approximately $10-25 \%$ (monthly fecundity $1-2 \%$ ) and so it might be reasonable to attempt non-ART treatment for a short period of time. Patients in Treatment Level IV have annual fecundity less than $10 \%$ and ART should be utilized as soon as possible, because very few pregnancies will occur without ART.

Tables 1 and 2 show that patients with an EFI score of 7 do not experience the same decrease in pregnancy rates to a Level III or IV as all other EFI scores. Interestingly, patients with an EFI score of 7 are the only group to persist with Level II treatment recommendations in year 3 . The reasons for the drop to Treatment Level I or II for EFI scores $0-6$ are probably different than for EFI scores 8-10. It seems reasonable that the low year 3 pregnancy rates for EFI scores of $0-6$ is a result of a reproductive system that has global issues and is generally not working well. Patients with an EFI score of 8-10 show a higher initial pregnancy rate with a more dramatic decrease in pregnancy rates by year 3 . This seems to reflect a reproductive system that overall is working fairly well but includes a segment of patients with an unknown and undiagnosed contributing factor to their infertility, one that often is nullified with ART. Patients with an EFI score of 7 seem to have a compromised but intact reproductive system that provides a relatively consistent, albeit reduced, fecundity over time.

For all patients, controlled ovarian stimulation with clomiphene citrate should be used for 3 to 6 months before ART unless the patient is in Level IV where ART is generally the most appropriate first line of treatment. COS should be used for more than 3 months only in young patients responding well to the medications. Recent studies have shown that, compared with IVF, gonadotropins and IUI treatment do not result in higher pregnancy rates, and also it takes longer to conceive, costs more money, and results in more multiple pregnancies [27•]. Therefore, gonadotropins and IUI generally should not be used but rather patients should go straight from clomiphene to ART.

Obviously many factors are considered when counseling an infertile couple about their options and recommended course of treatment. Referral or ART does not have to proceed on a fixed schedule. Indeed, it does appear pregnancies will continue to occur even after 1-2 years, although at a lower monthly fecundity. Even a relatively low monthly fecundity will over time result in a significant number of pregnancies. Some couples may wish to proceed with nonART pregnancy attempts over a long period of time or may never opt for ART treatment. Table 3 outlines a treatment algorithm, which provides the basis of general guideline
Table 2 Treatment levels and recommendations

\begin{tabular}{lll}
\hline Treatment level & $\begin{array}{l}\text { Monthly } \\
\text { fecundity }\end{array}$ & $\begin{array}{l}\text { Treatment } \\
\text { recommendation }\end{array}$ \\
\hline I & $>3 \%$ & Attempt non-ART conception for at least 1 year \\
II & $2-3 \%$ & Probable attempt non-ART conception, consider role of IVF \\
III & $1-2 \%$ & Probable IVF, refer to reproductive endocrinologist for fertility management \\
IV & $<1 \%$ & Refer to ART center for IVF \\
\hline
\end{tabular}


Table 3 EFI score and treatment level

\begin{tabular}{llll}
\hline EFI score & \multicolumn{2}{l}{ Treatment level } \\
\cline { 2 - 4 } All patients $(n=801)$ & Year 1 & Year 2 & Year 3 \\
\hline $0-3$ & IV & IV & IV \\
4 & III & IV & IV \\
5 & III & III & IV \\
6 & II & II & III \\
7 & I & II & II \\
8 & I & II & III \\
$9-10$ & I & I & IV \\
\hline
\end{tabular}

recommendations for treatment of women with endometriosisrelated fertility.

This analysis demonstrates additional ways to utilize the EFI for management of endometriosis patients. It is hoped this additional information will maximize its clinical usefulness and as a result provide the best chance of positively impacting the care provided to all endometriosis fertility patients (Table 4).

\section{Predicting Pregnancy Rate with ART in Endometriosis Patients}

The peritoneal lesions of stage III endometriosis, ovarian endometriomas, and deep infiltrating endometriosis all have been reported to independently affect fertility and ART success rates [28, 29].

The role and potential benefit of surgery both alone and in conjunction with ART has been debated. Several studies of surgical treatment alone have demonstrated a positive impact on both Stage I-II and Stage III-IV endometriosis [21••, 30, 31]. Ballester et al. recently published their findings and presented a nomogram providing precise information about ICSI-IVF success to be used as a guide for both couples and practitioners [32]. Clinical pregnancy rates for patients with and without deep infiltrating endometriosis were reported at $58 \%$ and $83 \%$ respectively, but the study had many limitations.

Darai et al. evaluated the effect of colorectal resection of endometriosis on fertility [33]. The 52 patients were randomized to laparoscopic or open surgery. Laparoscopic surgery resulted in a lower incidence of adhesion formation. The pregnancy rate of $39.3 \%$ was similar to that of other studies. Two thirds of spontaneous pregnancies in this study occurred in the first year and none occurred after 18 months. No patient older than age 35 years experienced spontaneous pregnancy.

\section{Conclusions}

A variety of endometriosis diagnostic tests, scoring systems, and treatment data outcome have been published recently. This information provides a working basis for constructing a rational and systematic approach to evaluation and treatment of endometriosis-related infertility patients. Treatment consideration should include availability of time to conceive, ovarian reserve, and the possibility to conceive by non-ART means.

The first step in counseling an endometriosis patient with potential fertility issues is evaluation of ovarian reserve. Early screening of ovarian reserve in younger women with advanced invasive endometriosis may be indicated even before the actual time of wanting to conceive. Women with a decreased ovarian reserve should consider ART as first-line therapy. Other factors, such as quality of sperm and uterine status, also need to be assessed and considered.

The endometriosis fertility index (EFI) provides the basis for predicting the pregnancy rate over 3 years and providing a simple visual graph for education of the physician and patient. A treatment algorithm derived from these data is presented. Infertility patients in whom ART is a potential treatment option generally should have started ART by the time they are 36 or 37 years old. The length of time endometriosis-

Table 4 Age-weighted endometriosis treatment algorithm

\begin{tabular}{|c|c|c|c|c|c|c|c|c|c|c|c|}
\hline \multicolumn{4}{|l|}{ 1st year } & \multicolumn{4}{|l|}{ 2nd year } & \multicolumn{4}{|l|}{ 3rd year } \\
\hline EFI score & $<34$ & $35-37$ & $>37$ & EFI score & $<34$ & $35-37$ & $>37$ & EFI score & $<34$ & $35-37$ & $>37$ \\
\hline $0-3$ & IV & IV & IV & $0-3$ & IV & IV & IV & $0-3$ & IV & IV & IV \\
\hline 4 & III & IV & IV & 4 & IV & IV & IV & 4 & IV & IV & IV \\
\hline 5 & III & IV & IV & 5 & III & IV & IV & 5 & IV & IV & IV \\
\hline 6 & II & III & IV & 6 & II & III & IV & 6 & III & IV & IV \\
\hline 7 & I & II & III & 7 & II & III & IV & 7 & II & III & IV \\
\hline 8 & I & II & III & 8 & II & III & IV & 8 & III & IV & IV \\
\hline $9-10$ & I & II & III & $9-10$ & I & II & III & $9-10$ & IV & IV & IV \\
\hline
\end{tabular}


related fertility patients should attempt pregnancy by non-ART methods following surgery for treatment of endometriosis can range from zero months to 2-3 years in very young patients, as elucidated by the EFI. Very few patients with access to IVF will wait much longer than a year. But for patients who do not have access to IVF, the EFI can give a realistic assessment of pregnancy rates for up to 3 years, after which very few pregnancies occur in any patients regardless of EFI score.

Based on this information, patients should undergo surgical treatment of endometriosis, if clinically indicated, by age 33 years to provide sufficient time for success in attaining pregnancy by non-ART. This approach avoids beginning the ART phase of fertility treatment, if needed, after the patient has entered into a period of DOR.

The EFI score can be used to create non-ART endometriosis treatment levels to help provide the patient and physician with increased understanding of the patient's current fertility potential and the prognosis for non-ART conception. Further research hopefully will help us to continue to improve the management of endometriosis infertility patients.

\section{Compliance with Ethics Guidelines}

Conflict of Interest Andrew S. Cook declares that he has no conflict of interest.

G. David Adamson is a consultant for Auxogyn and Sonoa Healthcare, has received honoraria from Best of ASRM/ESHRE and Women's Hospital of Texas, has received royalties from Cambridge University Press, and holds stock or stock options with Advanced Reproductive Care, Inc. and netMD.

Human and Animal Rights and Informed Consent This article does not contain any studies with human or animal subjects performed by any of the authors.

\section{References}

Papers of particular interest, published recently, have been highlighted as:

- Of importance

•- Of major importance

1. Buyuk E, Seifer DB, Younger J, Grazi RV, Lieman H. Random antiMüllerian hormone (AMH) is a predictor of ovarian response in women with elevated baseline early follicular follicle-stimulating hormone levels. Fertil Steril. 2011;95(7):2369-72.

2. - The FIGO Fertility Tool Box http://www.figo.org/news/resources/ FIGO_Fertility_Tool_Box. p. 41. The FIGO Fertility Tool Box provides a comprehensive overview of the management of infertility for all levels of healthcare providers in any clinical setting.

3. The Practice Committee of the American Society for Reproductive Medicine. American Society for Reproductive Medicine, Birmingham, Alabama. Age-related fertility decline: a committee opinion. Fertil Steril. 2008;90:S154-5.

4. Leridon $\mathrm{H}$. A new estimate of permanent sterility by age: sterility defined as the inability to conceive. Popul Stud (Camb). 2008;62:15-24.
5. Te Velde ER, Pearson PL. The variability of reproductive aging. Hum Reprod Update. 2002;8:141-54.

6. •- ASRM Practice Committee. Testing and interpreting measures of ovarian reserve: a committee opinion. Fertil Steril. 2012;98(6):140815. Assessment of ovarian reserve is a critical component of selecting the appropriate treatment from among those options available for any given patient. This document is written by a committee of experts who provide an unbiased overview of the complexity of ovarian reserve testing and how to interpret the results in the clinical setting.

7. Shah DK. Diminished ovarian reserve and endometriosis: insult upon injury. Semin Reprod Med. 2013;31:144-9.

8. Shebl O, Ebner T, Sommergruber M, Sir A, et al. Anti müllerian hormone serum levels in women with endometriosis: a case-control study. Gynecol Endocrinol. 2009;25(11):713-6.

9. The FIGO Fertility Tool Box http://www.figo.org/news/resources/ FIGO_Fertility Tool Box. p. 69.

10. The Practice Committee of the American Society for Reproductive Medicine in collaboration with the Society of Reproductive Surgeons. American Society for Reproductive Medicine, Birmingham, Alabama. Myomas and reproductive function. Fertil Steril. 2008;90:S125-30.

11. Verkauf BS. Myomectomy for fertility enhancement and preservation. Fertil Steril. 1992;58:1-15.

12. The Practice Committee of the American Society for Reproductive Medicine. American Society for Reproductive Medicine, Birmingham, Alabama. Pathogenesis, consequences and control of peritoneal adhesions in gynecologic surgery: a committee opinion. Fertil Steril. In press. https://www.asrm.org/uploadedFiles/ ASRM_Content/News and Publications/Practice_Guidelines/ Committee_Opinions/Ad̄hesions_2013.pdfmembers.pdf. Accessed online May 3, 2013.

13. The FIGO Fertility Tool Box http://www.figo.org/news/resources/ FIGO_Fertility_Tool_Box.pp. 55, 69.

14. •- The Practice Committee of the American Society for Reproductive Medicine. American Society for Reproductive Medicine, Birmingham, Alabama. The role of tubal surgery in the era of assisted reproductive technology. Fertil Steril. 2012;97:539-45. The development of assisted reproductive technologies has made many formerly performed reproductive surgeries either unnecessary or inappropriate because of unfavorable effectiveness, cost, time to pregnancy, risk, or other factors. Nevertheless, reproductive surgery is still a better option than ART for many patients because of superior outcomes and treatment of related problems, such as pain, especially true for endometriosis. This comprehensive and balanced document written by experts assesses the contemporary role of tubal surgery.

15. The FIGO Fertility Tool Box http://www.figo.org/news/resources/ FIGO_Fertility_Tool_Box.p. 44.

16. ASRM and SMRU. The Practice Committee of the American Society for Reproductive Medicine in collaboration with the Society for Male Reproduction and Urology. American Society for Reproductive Medicine. Birmingham, Alabama. Evaluation of the azoospermic male. Fertil Steril. 2008;90:S74-7.

17. The Practice Committee of the American Society for Reproductive Medicine. American Society for Reproductive Medicine, Birmingham, Alabama. Obesity and reproduction: an education bulletin. Fertil Steril. 2008;90:S121-4.

18. van der Steeg JW, Steures P, Eijkemans MJ, Habbema JD, Hompes PG, Burggraaff JM, et al. Obesity affects spontaneous pregnancy chances in subfertile, ovulatory women. Hum Reprod. 2008;23:324-8.

19. The Practice Committee of the American Society for Reproductive Medicine. American Society for Reproductive Medicine, Birmingham, Alabama. Smoking and infertility. Fertil Steril. 2008;90:S254-9.

20. - Barri PN, Coroleu B, Tur R, Barri-Soldevila PN, Rodriguez I. Endometriosis-associated infertility: surgery and IVF, a comprehensive therapeutic approach. Reprod BioMed Online. 2010;21:179-85. 
This study provides additional information regarding important factors that affect pregnancy rates that need to be considered in management decisions for endometriosis patients with infertility.

21. - Adamson GD, Pasta DJ. Endometriosis fertility index: the new, validated endometriosis staging system. Fertil Steril. 2010;94:160915. The Endometriosis Fertility Index (EFI) is the first validated endometriosis staging system that predicts non-ART pregnancy rates following the surgical diagnosis and treatment of endometriosis. This tool is easy to use by physicians and helps patients understand and participate in clinical decision making to decide how long to pursue non-ART attempts at conception before moving on to ART.

22. Tomassetti1, B. Geysenbergh, C. Meuleman, D. Timmerman, S. Fieuw, $\mathrm{S}$ and T. D'Hooghe External validation of the endometriosis fertility index (EFI) staging system for predicting non-ART pregnancy after endometriosis surgery. Hum Reprod. 2012;0(0):1-9.

23. Wei DM, Yu Q, Sun AJ, Tian QJ, et al. Relationship between endometriosis fertility index and pregnancies after laparoscopic surgery in endometriosis-associated infertility. Zhonghua $\mathrm{Fu}$ Chan Ke Za Zhi. 2011;46(11):806-8.

24. Yacoub A, Ferdinus C, Mourtialon P, et al. Is Endometriosis Fertility Index a good tool to predict pregnancy in patients with surgical documented endometriosis followed by ART Management? World Congress Endometriosis, Montpelier, France. Clinical Free Oral Communication S\#10-4. 7 September 2011.

25. D'Hooghe T, Hummelshoj L. Multi-disciplinary centres/networks of excellence for endometriosis management and research: a proposal. Hum Reprod. 2006;21(11):2743-8. Epub 2006 Sep 18.

26. Adamson GD, Hurd SJ, Pasta DJ, Rodriguez BD. Laparoscopic endometriosis treatment: is it better? Fertil Steril. 1993;59:35-44.
27. - Reindollar RH, Regan MM, Neuman PJ, et al. A randomized clinical trial to evaluate optimal treatment for unexplained infertility: the Fast Track and Standard Treatment (FASTT) Trial. Fertil Steril. 2010;94:888-99. This excellent, large, randomized trial provided evidence on which to base decisions regarding the duration and type of controlled ovarian stimulation before ART treatment.

28. Vercellini P, Barbara G, Buggio L, Frattaruolo MP, et al. Effect of patient selection on estimate of reproductive success after surgery for rectovaginal endometriosis: literature review. Reprod BioMed Online. 2012;24:389-95.

29. Douay-Hauser N, Yazbeck C, Walker F, Luton D, et al. Infertile women with deep and intraperitoneal endometriosis: comparison of fertility outcome according to the extent of surgery. J Minim Invasive Gynecol. 2011;18:622-8.

30. Adamson GD, Pasta DJ. Surgical treatment of endometriosisassociated infertility: meta-analysis compared with survival analysis. Am J Obstet Gynecol. 1994;171:1488-504. discussion 504-5.

31. Marcoux S, Maheux R, Berube S. Laparoscopic surgery in infertile women with minimal or mild endometriosis. Canadian Collaborative Group on Endometriosis. N Engl J Med. 1997;337:217-22.

32. Ballester M, Oppenheimer A, d'Argent M, Touboul C, et al. Nomogram to predict pregnancy rate after ICSI IVF cycle in patients with endometriosis. Hum Reprod. 2012;27(2):451-6. doi:10.1093/ humrep/der392. Epub 2011 Nov 23.

33. Darai E, Lesieur B, Dubernard G, Rouzier R, et al. Fertility after colorectal resection for endometriosis: results of a prospective study comparing laparoscopy with open surgery. Fertil Steril. 2011;95:1903-8. 